\title{
Association of Myosin Light Chain 2 (MLC 2) with Troponin-I and Ejection Fraction in patients with Acute Coronary Syndrome
}

\author{
Samira Anwar ${ }^{1}$, Mansura Khan ${ }^{2}$, Ashesh Kumar Chowdhury ${ }^{3}$
}

\begin{abstract}
Background: Acute coronary syndrome (ACS) is a major health problem and represents a large number of hospitalizations annually. Myosin light chain (MLC2)isasensitive early marker (within four hours) of ACS that can be used to confirm or exclude a diagnosis of acute cardiac condition. The present study was undertaken for rapid diagnosis ACS with the help of elevated MLC-2and its relation with Troponin I and ventricular ejection fraction.

Method: In this cross sectional study, 60 patients ofACS were enrolled from coronary care unit (CCU), BIRDEM as case and 25 apparently healthy people from cardiac point of view as control. Selection of patients was done according to the inclusion and exclusion criteria.MLC-2was measured(within 3-4 hours by ELISA method and level of ventricular dysfunction and troponin I (in ACS group) was taken from hospital records.

Results: The mean $\pm S E M$ of MLC-2 was higher in the ACS group $(88.02 \pm 18.0 \mathrm{pgm} / \mathrm{ml})$ compared to the healthy control group $(41.4 \pm 2.2 \mathrm{pgm} / \mathrm{ml})$ and difference was statistically significant $(p<0.05)$. The inverse correlation between $M C L-2$ and ejection fraction was highly significant $(r=-0.647, p=0.001)$. The correlation with MLC-2 and troponin I was also significant $(r=0.831, p=0.01)$.

Conclusion: Statistically significantMLC2level was found in the ACS group than the healthy control group. Positive correlation was also observed between MLC-2 with Troponin I and ventricular dysfunction. Therefore, MLC 2 may be used as a biomarker early diagnosis of ACS.
\end{abstract}

Key Words: ACS, MLC2, Troponin I, Ejection fraction.

\section{Introduction :}

Patients with acute coronary syndrome (ACS) may lead to excess morbidity and mortality that could be prevented with optimal treatment ${ }^{(1)}$. As ACS is becoming a significant burden on health care services in world and as well as Bangladesh, research should be done to identify the etiological factors and design realistic strategies to improve the early diagnosis and health care facilities. The World Health Organization (WHO) estimates 17.5 million peoplein 2012 diedand there will be about 20 million CVD deaths in 2015, accounting for 30-31 percent of all deaths worldwide ${ }^{(2)}$. Although low and middle income countries account for more than $80 \%$ of global deaths and $85 \%$ of global disability from CVD. Bangladesh probably has the highest rate of CVD of all south Asian countries ${ }^{(3)}$. As ACS is becoming a significant

1. Dr. Samira Anwar, Dept. of Immunology, Bangladesh Institute of Research and Rehabilitation in Diabetes, Endocrine and Metabolic Disorders (BIRDEM),

2. Dr. Mansura Khan, Assistant Professor, Dept. of Immunology, BIRDEM

3. Dr. Ashesh Kumar Chowdhury, Professor, Department of Immunology, BIRDEM

\section{Corresponding Author:}

Dr. Samira Anwar

Dept. of Immunology

Bangladesh Institute of Research and Rehabilitation in Diabetes,

Endocrine and Metabolic Disorders (BIRDEM),

Email: samira_rmizan@yahoo.com burden on health care services in world and as well as Bangladesh, research should be done to identify the etiological factors and design realistic strategies to improve the early diagnosis and health care facilities.

The early appearance of a marker released into the bloodstream soon after an injury may facilitate early diagnosis. The marker release should be in proportion to the extent of myocardial injury has led to the use of cardiac markers to estimate the size or severity of an injury ${ }^{(4)}$.Simple multimarker strategy that categorizes patients based on the number of elevated biomarkers at presentation allows risk stratification $^{(5)}$.

MLC2 (myosin light chain) appears to have a high diagnostic validity for the assessment of minimal myocardial damages ${ }^{(6)}$. MLC concentration may be used for mortality prediction because in these patients increased MLC2 levels may due to degeneration of myofibrils leads to myocardial dysfunction. Moreover, these structural changes may acceleratedys function of ventricles more and enhance the filling pressure. Another reason may be MLC gene over expression. This factor may lead to an over synthesis of protein in myocardial cells and its transfer into circulation. Such transfer is associated with an impaired structure of cardiomyocytes. Patients with increased MLC2 may reflect myocytic stress, impaired integrity of myocytic membrane, or impaired structure of cardiomyocytes which leads high mortality ${ }^{(6-7)}$.Interpretation of cardiac biomarkers data in ACS and heart failure provides better clinical guidance ${ }^{(8)}$. Infract size ,clinical severity and prognosis have been reported to correlate with peak MLC level in combination with other 
cardiac markers provide more useful information and have a great value in risk stratification of ACS patients ${ }^{(9-10)}$.

To the best of our knowledge, no such study with MLC 2 has been carried out in Bangladesh. Therefore, we understand the study to see the association of MLC-2 with establish ACS marker TroponinI and with ejection fraction.

\section{Materials and Methods:}

This cross sectional study was conducted in the Department of Immunology, Bangladesh Institute of Research and Rehabilitation in Diabetes, Endocrine and Metabolic Disorders (BIRDEM) General Hospital, and Dhaka from July, 2014 to June, 2015. A total number of 60 subjects having chest pain and ECG finding other than normal were enrolled ascase from the coronary care unit (CCU) of the department of cardiology, BIRDEM General Hospital and 25 subjects having normal ECG with no cardiac complain were selected fromthe out-patient department of same hospital. CKD (Chronic kidney disease) or ESRF (end stage renal failure) patients, post-operative surgical patients having ACS, patients having septicemia and prior coronary artery bypass surgery subjects were excluded in this study.

Taking all aseptic precaution $5 \mathrm{ml}$ of blood sample was collected within 3-4 hours by using sterile disposable syringe and the blood sample was transferred carefully into sterile test tube. After collection in test tube the blood was allowed to clot at room temperature and serum was separated from the clot as soon as possible by centrifugation at $1000 \mathrm{rcf}$ for 15 to 20 minutes. The samples were kept at $-70^{\circ} \mathrm{C}$ till tested. Prior to the test, all samples were bought be at room temperature. Samples were vortexes before use as per recommendation of the kit instruction. Test was done by enzyme linked immunoassay (ELISA) kit from Biofarma, USA and result was calculated by MULTISCAN FC (thermo scientific, USA). The results of troponin I of ACS group (Immulite 2000, SIEMENS, Germany) and ejection fraction of study subjects was taken from hospital records. The study was approved by the ethical Review Committee of Diabetic Association of Bangladesh.

\section{Results:}

Total 85 subjects were enrolled in this cross sectional study. 60 of them were patients of acute coronary syndrome (ACS) and 25 subjects were healthy from cardiac point . The study subjects were divided into four age groups. Mean age \pm SEM of ACS group and healthy control group were 54.6 \pm 1.1 years and 52.6 \pm 2.0 years respectively. Most of the ACS patient belonged to 51-60 year's (43.3\%) age group. Both of the groups matched according to the mean age distribution.65\% was male and $35 \%$ was female in ACS group, whereas $60 \%$ was male and $40 \%$ was female in the healthy control group. Both the groups matched according to sex distribution $(\mathrm{p}>0.05)$ (table-I).

Table I: Age and sex distribution of the study subjects

\begin{tabular}{|c|c|c|c|c|c|c|c|c|c|}
\hline \multirow{2}{*}{$\begin{array}{l}\text { Age } \\
\text { (in } \\
\text { year) }\end{array}$} & \multicolumn{4}{|c|}{$\begin{array}{c}\text { ACS Group } \\
n=60\end{array}$} & \multicolumn{4}{|c|}{$\begin{array}{l}\text { Non ACS (healthy control ) Group } \\
\qquad \mathrm{n}=25\end{array}$} & $\mathrm{p}$ value \\
\hline & $\begin{array}{c}\text { Number of } \\
\text { subjects }\end{array}$ & $\begin{array}{c}\text { Mean } \\
\pm \\
\text { SEM }\end{array}$ & \multicolumn{2}{|c|}{ Sex } & $\begin{array}{l}\text { Number of } \\
\text { subjects }\end{array}$ & $\begin{array}{l}\text { Mean } \\
\pm \text { SEM }\end{array}$ & \multicolumn{2}{|c|}{ Sex } & $>0.05$ \\
\hline $30-40$ & $05(08.3 \%)$ & \multirow{4}{*}{$\begin{array}{l}54.642 \\
\pm 1.1\end{array}$} & Male & Female & $\begin{array}{l}02 \\
(08.0 \%)\end{array}$ & \multirow{4}{*}{$\begin{array}{c}52.6 \pm \\
2.0\end{array}$} & Male & Female & \\
\hline $41-50$ & $19(31.6 \%)$ & & \multirow[t]{3}{*}{$(65 \%)$} & \multirow[t]{3}{*}{$\begin{array}{c}23 \\
(35 \%)\end{array}$} & $\begin{array}{c}09 \\
(36.0 \%)\end{array}$ & & \multirow[t]{3}{*}{$\begin{array}{c}15 \\
(60 \%)\end{array}$} & \multirow[t]{3}{*}{$\begin{array}{c}10 \\
(40 \%)\end{array}$} & \\
\hline $51-60$ & $26(43.3 \%)$ & & & & $10(40.0)$ & & & & \\
\hline$\geq 61$ & $10(16.6 \%)$ & & & & $04(16.0)$ & & & & \\
\hline Range & \multicolumn{4}{|c|}{$40-75$} & \multicolumn{4}{|c|}{$40-78$} & \\
\hline
\end{tabular}

*Difference between ages in two groups was calculated by t-test

\section{Level of Myosin light chain(MLC2) in study subjects:}

The mean MLC2 was higher in ACS group then the healthy control group. The mean MLC in ACS group was $88.0 \pm 18.0 \mathrm{pg} / \mathrm{dl}$ and in the healthy non ACS group was $41.4 \pm 2.2 \mathrm{pg} / \mathrm{dl}$. The difference between the two means were statistically significant $(\mathrm{p}<0.05)$. The mean ejection fraction was lower in ACS group than the healthy control group. The mean ejection fraction \pm SEM in ACS group was $48.9 \pm 1.6 \%$ and in the healthy non ACS group was $69.1 \pm \mathbf{1 . 5} \%$. The difference between the two means were statistically significant $(\mathrm{p}<0.05)($ table-II). 
Table II: MLC 2 level of the study subjects

\begin{tabular}{|c|c|c|c|c|c|c|}
\hline \multirow{2}{*}{$\mathrm{pg} / \mathrm{dl}$} & \multicolumn{3}{|c|}{ MLC 2 level } & \multicolumn{3}{c|}{ Ejection fraction } \\
\cline { 2 - 7 } & $\begin{array}{c}\text { ACS Group } \\
(\mathrm{n}=60)\end{array}$ & $\begin{array}{c}\text { Non ACS } \\
\text { group } \\
(\mathrm{n}=25)\end{array}$ & $\begin{array}{c}\mathrm{p} \\
\text { value }\end{array}$ & $\begin{array}{c}\text { ACS Group } \\
(\mathrm{n}=60)\end{array}$ & $\begin{array}{c}\text { Non ACS } \\
\text { group }(\mathrm{n}=25)\end{array}$ & $\mathrm{p}$ value \\
\hline Mean \pm SEM & $88.0 \pm 18.0$ & $41.4 \pm 2.2$ & $<0.05$ & $48.9 \pm 1.6$ & $69.1 \pm 1.5$ & $<0.01$ \\
\hline Range & $52.0-124.0$ & $36.6-46.1$ & & $45.6-52.1$ & $65.9-72.3$ & \\
\hline
\end{tabular}

*p value was calculated by t-test.

Relation between ejection fraction and MLC2 in study subjects:

Aninverse relation between MLC2 and ejection fraction was observed between ACS and healthy group. Analysis done by
Pearson's correlation test and $r$ value (table-III) confirms correlation between MLC2 and ejection fraction.

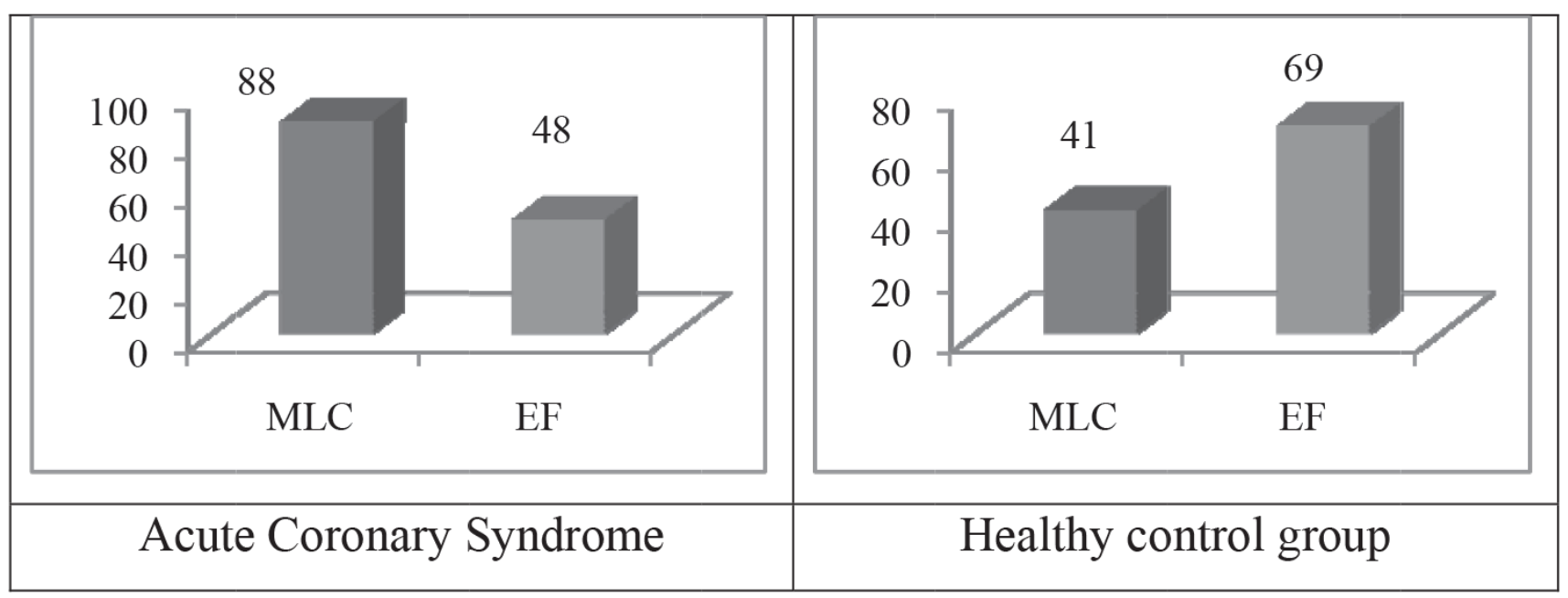

Figure I : Relation between ejection fraction and MLC2

\section{Relation of MLC 2 with ejection fraction and Troponin I in ACS group:}

To evaluate the relation of MLC 2 with ejection fraction and troponin I,Pearson correlation test was done which measure the strength of relationship between two variables by $r$ value and express on scatter plot.Mean troponin I in ACS group was $17.5 \pm 3.2 \mathrm{pg} / \mathrm{dl}$. Calculated $\mathrm{r}$ value was 0.83 which wassignificant $(p=0.01)$. There was a positive relation between MLC2 and troponin I. In majority of cases, when MLC2 increased, troponin I also increased in the same pattern (table III, figure II). MLC2 level was inversely related to ejection fraction as indicated by negative $r$ value
$(-0.647)$ which was also significant $(p<0.001)$. That means patient having more MLC2 had less ventricular ejection fraction which leads to more dysfunction.

Table III: Relation of myosin light chain 2 tropnin I and ejection fraction in ACS group

\begin{tabular}{lcc}
\hline Variables & r-value & P value \\
\hline Ejection fraction & -0.647 & 0.001 \\
Troponin I & 0.831 & 0.01 \\
\hline
\end{tabular}

*r-value was calculated by Pearson's correlation test. 

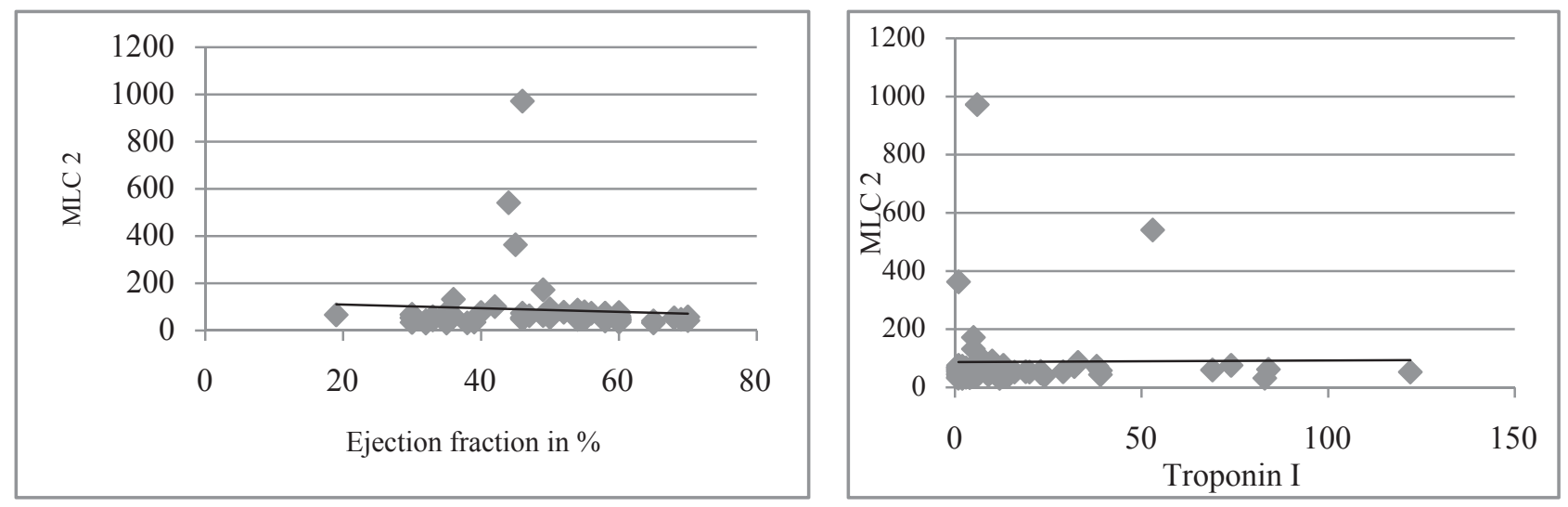

Figure II: Relation between MLC2 with Ejection Fraction and troponin I

\section{Discussion:}

Bangladesh demonstrated a dramatic increase in CVD from 1986 to $2006^{(3)}$. Recent evidence indicates that myocardial cell necrosis following artery occlusion can be reduced by varieties of therapeutic intervention ${ }^{(5)}$. First step of this procedure is early diagnosis.

The structure of sarcomere play a very important role in the pathogenesis of ischemic myocardial damage ${ }^{(8)}$. Thick and thin filaments are very important parts of sarcomere. So any changes of activities of these thickfilaments mediate tissue damage leads increase MLC2 concentration ${ }^{(9)}$. Increase levels of myosin light chain in serum indicates myocardial damage. Moreover, the initial ECG of a patient with ACS does not always reveal ST segment elevation or Q wave changes. MLC, a more recently developed cardiac marker, may be able to detect as soon as three hours after onset of ischemia. It could be therefore regarded one of the earliest biomarker available $^{(10)}$. One of the ten cases of heart attack victim die later from major damage to the heart muscle or suffer additional damage at later time. So it is essential to be detected substantially damage before the onset of irreversible damage. MLC can be used to confirm or exclude a diagnosis of acute coronary syndrome for monitoring of a recurrent infraction. The magnitude of increase in MLC has also demonstrated a good correlation with the severity of the infarction $^{(8,10)}$

Multi-marker approaches to risk stratification in ACS are also very important. Relationship between individual markers with other markers makes differ in treatment. Evidence based management is possible. It also helps in early clinical decision making ${ }^{(10-11)}$. Our second objective was to correlate MLC with ventricular dysfunction and troponin I for risk stratification. Literature survey indicated that no work has been done or reported on this biomarker and also multimarker strategies in cardiac patients in Bangladesh. This study was done considering the recent huge health burden of the cardiovascular diseases in Bangladesh. As such, it may offer some substantial guideline to formulate a better follow up and management protocol for acute coronary patients.
In the present cross-sectionalstudy, the total population of case group consisted of $65 \%$ male and $35 \%$ female and for control group; it was $60 \%$ male and $40 \%$ female. In both case sex wise male populations were roughly same. No significant gender difference was seen between these two groups.

We noted a significant difference between the levels of MLC2 in ACS group compared to non ACS group. The mean \pm SEM of MLC-2 was $88.02 \pm 18.0 \mathrm{pg} / \mathrm{ml}$ in case group and $41.4 \pm$ $2.2 \mathrm{pg} / \mathrm{ml}$ in control group. MLC 2 was significantly higher in ACS group. The difference between the two means where statistically significant $(p<0.05)$. The similar results were found by other researchers ${ }^{(11)}$.

It has been long establish that reduce ventricular ejection fraction is a significant indicator of cardiac dysfunction. Our study showed that the mean \pm SEM of ejection fraction was $48.90 \pm 1.61 \%$ in case group and $69.12 \pm 1.54 \%$ in control group. Ejection fraction was significantly higher in control group. There was a inverse relation between MLC 2 with ventricular dysfunction ( $\mathrm{r}$-0.647). Relation between ventricular dysfunction with MLC2 was statistically significant $(p<0.001)$. Our results were in accordance with study done by Farman et al. (2011)which assessed adverse correlation of MLC2 with ventricular ejection fraction ${ }^{(12)}$.

Troponin I is the marker of choice in ACS because of their superior sensitivity and specificity.The European society of cardiology support additional investigation with cardiac troponin I that occurs during reversible cardiac injury. In our study, we found significant positive relation between MLC2 and Troponin I $(r=0.831)$ and it was also statistically significant $(p<0.01)$. Another study by Graham et. al. (2003)analyzed the significant of study MLC2 with Troponin $\mathrm{I}^{13}$. The study also proved that MLC to be better predictor for monitoring acute coronary syndrome.

The 2012 guidelines from the Fifth Joint Task Force (JTF) of the European Societies on Cardiovascular Disease allows more evidence-based recommendations to be adapted to the needs of clinical practice. Myosin light chain contributes in evidence based management of myocardial damages. Thus the 
detection of circulating cardiac myosin light chains enables one to identify a subgroup of patients with angina at rest having more severe coronary artery disease with a worse outcome ${ }^{(14)}$.

Moreover through different correlation among cardiac markers we can judge and implement the best effective procedure of cardiac treatment.

\section{Conclusion:}

The present study showed that the mean myosin light chain 2 (MLC2) was higher in the acute coronary syndrome (ACS) group than the healthy control group and the differences were statistically significant. Positive correlation between MLC2 withTroponin I and ventricular dysfunction was also found. Therefore, MLC2 may be used as a biomarker to confirm diagnosis of acute coronary syndrome in conjunction with troponin I as it appears within 4 hours of myocardial damage. However, multi-centric study with large samples may be carried out to validate our result.

\section{References:}

1. Beaglehole $\mathrm{R}$ and Bonita R.Global Public Health:scorecard. Lancet2008;372(9654): 1988-1996.

2. Cardiovascular diseases(CVDs) Fact Sheet Reviewed onSeptember 2016. World health organization.accessed on 8.12.16 fromhttp://www.who.int/mediacentre/factsheets/fs317

3. Islam A.K.M. and A.A.S. Majumder. Coronary artery disease in Bangladesh: A review. Indian Heart Journal2013;65(4): 424-435.

4. Goto Y, Takase H, Toriyama T, Sugiura T, Sato K, Ueda R, and Dohi Y. Circulating concentrations of cardiac proteions indicate the severity of congestive heart failure. Heart2003;89(11):1303-1307.
5. Amodio G, Antonelli G, Di Serio F. Cardiac biomarkers in acute coronary syndromes: a review. Current Vascular Pharmacology2010;8(3):388-393.

6. Daniel F. The Slow Cardiac Myosin Regulatory Light Chain in Heart Failure.Clinical Cardiology2011;34 (1):10-11.

7. Hansen MS, Stanton EB, and Gawad Y. Relation of Circulationg Cardiac Myosin Light Chain Isoform in Stable Severe Congestive Heart Failure to Survival and Treatment WithFlosequinan. American Journal of Cardiology2002;90(9): 969-973.

8. Robert E. Using BNP to Diagnose manage, and Treat heart failure, Cleveland Clinic Journal of Medicine2003;70(4):333-336.

9. Li Y, Gang W, Qizhu T and wang H. Slow cardiac myosin regulatory light chain 2 (myl2) was down-expressed in chronic heart failure patients. Clinical Cardiology2010;34(1):30-34.

10. Kemp M., Donovan J, Higham H. and J. Hooper.Biochemical markers of myocardial injury. British Journal of Anaesthesia2004;93(1) : 63-73.

11. Richard LM andDaniel PF. Myosin Light Chain 2 into the Mainstream of Cardiac Development and Contractility. Circulation Research2006;99:225-227.

12. Farman G., Gore D, Allen E, Schoenfelt K, Irving T, and Tombe Pieter P . Myosin head orientation: a structural determinant for the Frank-Starling relationship. AmericanJournal ofPhysiology, Heart and Circulatory Physiology 2011;300 (6):2155-2160.

13. Graham S., Pamela T, Delana W, Lorraine H, Ning Z, William C. and Antoinette M.. The relative utility of cardiac troponin i, creatine kinase-mbmass, and myosin light chain-1 in the long-term risk stratification of patients with chest pain. Clinical Cardiology2003; 26(3): 147-152.

14. SzczesnaD, Hernandez O, Jones M, and Guzman G,. Myosin essential light chain in health and disease, American Journal of Physiology, Heart and Circulatory Physiology 2006;292:1643-1654. 